\title{
Low Platelet Count
}

National Cancer Institute

\section{Source}

National Cancer Institute. Low Platelet Count. NCI Thesaurus. Code C162108.

A lower than average level of platelets in a sample. 